\title{
ON SYMMETRY GROUPS OF YANG-MILLS AND SELF-DUAL YANG-MILLS EQUATIONS
}

\author{
(Presented by P. Kard)
}

The problem of finding groups of point transformations admissible by Yang-Mills equations and some forms of self-dual Yang-Mills equations in four-dimensional Euclidean space, is considered. The explicit form of basic generators for such groups is found. Some conserved quantities are obtained.

\section{Introduction}

It is well known that symmetry plays an important role in physics. There is a connection between symmetry groups and conserved quantities of a differential equation. The theory of group properties of differential equations worked out by S. Lie $\left[{ }^{1,2}\right]$, F. Engel $\left[{ }^{2}\right]$, A. Tresse $\left[{ }^{3}\right]$, E. Vessiot $\left[{ }^{4}\right]$, A. Cohen $\left[{ }^{5}\right]$, L. V. Ovsyannikov $\left[{ }^{6}\right]$ and others, has several applications in mechanics and other branches of physics $\left[{ }^{7-11}\right]$.

Here we call the reader's attention to some field equations interesting from the viewpoint of particle physics, such as the Yang-Mills and the so-called self-dual Yang-Mills equations.

Our aim is to find their symmetry groups as well as their trivial conservation laws. This forms a continuation of the investigation of field equations which we started with two-dimensional equations [ $\left.{ }^{12}\right]$ and then carried on with four dimensional ones. Detailed calculations are mostly omitted.

\section{Yang-Mills equations}

In 1954 C. N. Yang and R. L. Mills presented [ $\left.{ }^{13}\right]$ the local gauge invariance principle involving an isotopic spin. Compared to electrodynamics, a non-abelian symmetry was introduced.

The Yang-Mills equations are

$$
\partial_{\mu} F_{\mu v}-\left[A_{\mu}, F_{\mu v}\right]=0
$$

with the Lagrangian*

$$
囚=-\frac{1}{4} F_{\mu v}^{a} F_{\mu v}^{a}
$$

Here

$$
\begin{gathered}
F_{\mu v}=F_{\mu v}^{a} T^{a}=A_{\mu, v}-A_{v, \mu}+\left[A_{\mu}, A_{v}\right], \\
A_{\mu}=A_{\mu}^{a} T^{a}, \quad A_{\mu, v} \equiv \frac{\partial A_{\mu}}{\partial x_{v}} .
\end{gathered}
$$

* Here the sum convention is observed. The Greek indices take values from 1 to 4 , the Latin ones - from 1 to 3 . 
The matrices $T^{a}$ satisfy the conditions

$$
\begin{aligned}
& {\left[T^{a}, T^{b}\right]=t^{a b c} T^{c},} \\
& \operatorname{tr}\left[T^{a}, T^{b}\right]=-2 \delta^{a b},
\end{aligned}
$$

where the structure coefficients $t^{a b c}$ are completely antisymmetric.

We study the Yang-Mills equations in the Lorentz gauge:

$$
A_{\mu, \mu}=0,
$$

in which equations (1) take the form:

$$
\omega_{v} \equiv T^{c}\left[A_{v, \mu \mu}^{c}+t^{a b c} A_{\mu}^{a}\left(A_{\mu, v}^{b}-2 A_{v, \mu}^{b}+A_{\mu}^{k} A_{v}^{g} t^{k g b}\right)\right]=0 .
$$

We have found the algebra of the point transformation group admissible by the system of differential equations $\omega_{v}=0$, following the technique worked out by S. Lie $\left[{ }^{1-2}\right]$ and developed further by L. V. Ovsyannikov $\left[{ }^{6}\right]$, N. H. Ibragimov $\left[{ }^{1,8}\right]$, S. A. Vladimirov $\left[{ }^{9}\right]$ and others. This algebra is presented by generators

$$
X=\xi^{\mu}(x, A) \frac{\partial}{\partial x^{\mu}}+\eta^{c}(x, A) \frac{\partial}{\partial A_{\alpha}^{c}} .
$$

Coefficients $\xi^{\mu}$ and $\eta^{c}$ are determined by the equation

$$
\left.\widetilde{\widetilde{X}}_{\omega_{v}}\right|_{\omega_{v}=0}=0 \text {, }
$$

where

$$
\widetilde{\widetilde{X}}=X+\tilde{\zeta}_{\mu}^{c} \frac{\partial}{\partial p_{\mu}^{\alpha}}+\tilde{\sigma}_{\mu v}^{c} \frac{\partial}{\partial r_{\mu v}^{c}}
$$

is the prolonged generator. The new coefficients $\zeta_{\mu}^{c}$ and ${ }_{\sigma}^{c} \alpha$ depend on the previous ones in the following way:

$$
\begin{aligned}
& \zeta_{\mu}^{c}=D_{\mu} \eta^{\alpha}-p_{v}^{\alpha} D_{\mu} \xi^{v}, \\
& { }^{c} \alpha=\widetilde{D}_{\mu \nu}^{c} \zeta_{v}^{\alpha}-\stackrel{c}{r}_{\mu \sigma}^{\alpha} D_{v} \xi^{\sigma},
\end{aligned}
$$

where

$$
\begin{aligned}
& D_{\mu} \equiv \frac{\partial}{\partial x^{\mu}}+\stackrel{a}{p_{\mu}^{\alpha}} \cdot \frac{\partial}{\partial A_{\alpha}}, \quad \widetilde{D}_{\mu} \equiv D_{\mu}+\stackrel{a}{r}_{\mu \nu}^{a} \frac{\partial}{\partial p_{v}^{\alpha}}, \\
& \stackrel{a}{p_{\mu}^{\alpha}} \equiv \frac{\partial A_{\alpha}^{a}}{\partial x^{\mu}}, \quad{ }_{\mu \nu}^{a} \equiv \frac{\partial^{2} A_{\alpha}^{a}}{\partial x^{\mu} \partial x^{v}} .
\end{aligned}
$$

In the case of Yang-Mills equations, the system of determining equations is the following:

$$
\begin{aligned}
& \left.\widetilde{\widetilde{X}}_{(0 v}\right|_{(2)(3)}=0, \\
& \widetilde{\widetilde{X}}_{\left.p_{\mu}^{\mu}\right|_{(2)(3)}=0}
\end{aligned}
$$


or, in detail,

$$
\begin{aligned}
& \left.\stackrel{c}{\sigma_{\mu \mu}^{v}}+t^{a b c}\left[\stackrel{a}{\eta^{\mu}} \stackrel{b}{A^{\mu}{ }_{, v}}-2 \stackrel{b}{A^{v}}{ }_{, \mu}+\stackrel{k}{A^{\mu}} \stackrel{g}{A^{v}} t^{h g b}\right)+\stackrel{a}{A^{\mu}} \stackrel{b}{\left(\zeta_{v}^{\mu}\right.}-2 \stackrel{b}{\zeta_{\mu}^{v}}\right)+ \\
& \left.\left.+t^{k g b} \stackrel{a}{A} \stackrel{k}{\mu}^{\left(\eta^{\mu} \stackrel{g}{A}\right.}{ }^{v}+\stackrel{g}{\eta^{v}}{ }^{v} \stackrel{k}{A}^{\mu}\right)\right]\left.\right|_{(2)(3)}=0, \\
& \left.\check{\zeta}_{\mu}^{c}\right|_{(2)(3)}=0 \text {. }
\end{aligned}
$$

First, inserting in (4) indentities (3) and $r_{\mu \alpha}^{\mu}=0$ (the latter is a consequence of the Lorentz gauge condition (2)) and secondly, equating the coefficients at all remaining independent variables to zero, we get the solutions of the system (4)

$$
\xi_{\mu}=a_{\mu v} x_{v}+d \cdot x_{\mu}+c_{\mu}, \quad \stackrel{c}{\eta_{\mu}}=a_{\mu v} \stackrel{\mathrm{c}}{A_{v}}-d \cdot \stackrel{c}{A_{\mu}}+\varepsilon_{c \alpha} \stackrel{a}{A}_{\mu}
$$

where $a_{\mu v}=-a_{v \mu}, \varepsilon_{a b}=-\varepsilon_{b a}, c_{\mu}, d$ are constants. One can see that the generators of the symmetry group of Yang-Mills equations (3) in the Lorentz gauge (2) are:

where

$$
\begin{array}{ll}
X_{\mu}=\frac{\partial}{\partial x_{\mu}}, & X_{\mu v}=Z_{\mu v}+Y_{\mu v}, \\
X=x_{\mu} \frac{\partial}{\partial x_{\mu}}-\stackrel{c}{A}_{\mu} \frac{\partial}{\partial A_{\mu}^{c}}, . & X_{a b}=\stackrel{a}{A}_{\mu} \frac{\partial}{\partial A_{\mu}^{b}}-\stackrel{b}{A}_{\mu} \frac{\partial}{\partial A_{\mu}^{a}}
\end{array}
$$

$$
Z_{\mu \nu}=x_{\mu} \frac{\partial}{\partial x_{v}}-x_{v} \frac{\partial}{\partial x_{\mu}}, \quad Y_{\mu \nu}=\stackrel{\mathrm{A}}{\mu}_{\mu} \frac{\partial}{\partial \AA_{v}^{\mathrm{c}}}-\stackrel{\mathrm{c}}{A_{v}} \frac{\partial}{\partial \AA_{\mu}^{\mathrm{c}}}
$$

The rotation generators $X_{a b}$ form a gauge group $S O(3) \simeq S U(2)$. It is to be noted that the Yang-Mills equations without the gauge conditions admit a special conformal transformation

$$
X=\left(c_{\rho} x_{\rho} x_{\mu}-\frac{1}{2} c_{\mu} x_{\rho}^{2}\right) \frac{\partial}{\partial x_{\mu}}+\left[c_{\rho}\left(x_{\mu} \stackrel{c}{A_{\rho}}-x_{\rho} \stackrel{c}{A}_{\mu}\right)-c_{\mu} x_{\rho} \stackrel{c}{A}_{\rho}\right] \frac{\partial}{\partial \AA_{\mu}^{c}}
$$

which is in accordance with $\left[{ }^{14}\right]$.

\section{Self-dual Yang-Mills equations and related field equations}

Next, we find generators of the symmetry group admissible by self-dual Yang-Mills equations

$$
F_{\mu v}=\frac{1}{2} \varepsilon_{\mu \nu \alpha \beta} F_{\alpha \beta}
$$

where $F_{\mu v}=A_{\mu, v}-A_{v, \mu}+\left[A_{\mu}, A_{v}\right]$ and $A_{\mu}=-\frac{i}{2} \sigma_{a} A_{\mu} . \sigma_{a}$ are the Pauli matrices and $A_{\mu}^{a}$ are the self-dual gauge potentials.

In fact, the solutions of equations (6) also satisfy. Yang-Mills equations. They have the so-called instanton solutions $\left[{ }^{15}\right]$.

Using the method described above, it can be shown that the equations (6) are invariant under the transformation group represented by the generators 


$$
X_{\mu}=\frac{\partial}{\partial x^{\mu}}, \quad X_{\mu v}=\bar{Z}_{\mu v}+\dot{Y}_{\mu v}, \quad X=x^{\mu} \frac{\partial}{\partial x^{\mu}}-\stackrel{c}{A}^{\alpha} \frac{\partial}{\partial A_{\alpha}^{c}},
$$

which form the Weyl algebra. Introducing the new variables

$$
\begin{array}{ll}
y=\left(x_{1}+i x_{2}\right) / 2, & \bar{y}=\left(x_{1}-i x_{2}\right) / 2, \\
z=\left(x_{3}-i x_{4}\right) / 2, & \bar{z}=\left(x_{3}+i x_{4}\right) / 2
\end{array}
$$

one obtains for the equations (6) the form

$$
F_{y z}=0, \quad F_{\overline{y z}}=0, \quad F_{y y}+F_{z \bar{z}}=0 .
$$

It is known $\left[{ }^{15}\right]$ that the solution of the first two equations is

$$
\begin{array}{ll}
A_{y}=D^{-1} D_{, y}, & A_{\bar{y}}=-D_{, y}^{ \pm} D^{+-1}, \\
A_{z}=D^{-1} D_{, z}, & A_{\bar{z}}=-D_{\frac{ \pm}{, z}}^{ \pm} D^{+-1},
\end{array}
$$

where $D \in S L(2, C)$.

The third equation for the variable $\chi=D D^{+}$has the following form

$$
\left(\chi^{-1} \cdot \chi_{, y}\right)_{, y}+\left(\chi^{-1} \cdot \chi_{, z}\right)_{, ;}=0,
$$

where

$$
\chi \in S L(2, C) .
$$

First, we search for variable $\chi$ in the form

$$
\chi=q^{0} I+q^{a} \sigma_{a}=q^{\alpha} \sigma_{\alpha} \quad\left(\sigma^{0} \equiv I\right),
$$

then one can get for $q^{\alpha}$ the equation

$$
\begin{gathered}
q_{, y y}^{\alpha}+q_{, z z}^{\alpha}+q^{\alpha}\left(q_{, y}^{\beta} q_{, y}^{\beta}+q_{, z}^{\beta} q_{, z}^{\beta}\right)+ \\
+i \varepsilon^{\alpha \beta \gamma \delta} q^{\beta}\left(q_{, y}^{\gamma} q_{, y}^{\delta}+q_{, z}^{\gamma} q_{, z}^{\delta}\right)=0 .
\end{gathered}
$$

In terms of the former variables $x_{\mu}$, this equation has the form

$$
q_{, \mu \mu}^{\alpha}+q^{\alpha}\left(q_{, \mu}^{\beta} q_{, \mu}^{\beta}\right)+2 \varepsilon^{\alpha \beta \gamma \delta} q^{\beta}\left(q_{, 1}^{\gamma} q_{, 2}^{\delta}-q_{, 3}^{\nu} q_{, 4}^{\delta}\right)=0 .
$$

The symmetry group generators of this equation form a space $L_{15}$ :

$$
\begin{aligned}
& X_{\mu}=\frac{\partial}{\partial x_{\mu}}, \\
& X_{5}=x^{\mu} \frac{\partial}{\partial x^{\mu}}, \\
& X_{6}=x_{1} \frac{\partial}{\partial x_{2}}-x_{2} \frac{\partial}{\partial x_{1}}, \\
& X_{7}=x_{3} \frac{\partial}{\partial x_{4}}-x_{4} \frac{\partial}{\partial x_{3}}, \\
& X_{8}=x_{1} \frac{\partial}{\partial x_{3}}-x_{3} \frac{\partial}{\partial x_{1}}-x_{2} \frac{\partial}{\partial x_{4}}+x_{4} \frac{\partial}{\partial x_{2}},
\end{aligned}
$$




$$
\begin{aligned}
& X_{9}=x_{1} \frac{\partial}{\partial x_{4}}-x_{4} \frac{\partial}{\partial x_{1}}+x_{2} \frac{\partial}{\partial x_{3}}-x_{3} \frac{\partial}{\partial x_{2}}, \\
& X_{\alpha \beta}=q_{\alpha} \frac{\partial}{\partial q_{\beta}}-q_{\beta} \frac{\partial}{\partial q_{\alpha}} .
\end{aligned}
$$

Compared to generators (7), the Lorentz-invariance gets lost here. Generators $Z_{\mu \nu}$ in (7) correspond to generators $X_{6}-X_{9}$ in (12). A new symmetry appears - dilatation $X_{5}$.

Secondly, in equation (9) variable $\chi$ can be parametrised by the use of Poincaré coordinates $\left[{ }^{15}\right]$ :

$$
\chi=\frac{1}{u^{1}}\left(\begin{array}{cc}
1 & u^{3} \\
u^{2} & \left(u^{1}\right)^{2}+u^{2} u^{3}
\end{array}\right),
$$

where $u^{2} \equiv \overline{u^{3}}$. Then one obtains the system of Yang equations $\left[{ }^{16}\right]$ :

$$
\begin{aligned}
& u^{1}\left(u_{, y \bar{y}}^{1}+u_{, z \bar{z}}^{1}\right)-u_{, y}^{1} u_{, y}^{1}-u_{, z}^{1} u_{, z}^{1}+u_{, y}^{2} u_{\frac{1}{y}}^{3}+u_{, z}^{2} u_{, \bar{z}}^{3}=0, \\
& u^{1}\left(u_{, y \bar{y}}^{2}+u_{, z \bar{z}}^{2}\right)-2 u_{, y}^{2} u_{, y}^{1}-2 u_{, z}^{2} u \frac{1}{, z}=0 \\
& u^{1}\left(u_{, y y}^{3}+u_{, z \bar{z}}^{3}\right)-2 u_{, y}^{1} u_{\frac{1}{y}}^{3}-2 u_{, z}^{1} u_{, \bar{z}}^{3-}=0
\end{aligned}
$$

which is equivalent to the system of Ernst equations $\left[{ }^{17-19}\right]$. The symmetry group of the system (13) is the following:

$$
\begin{aligned}
& X_{\mu}=\frac{\partial}{\partial x_{\mu}} \\
& X_{5}=x^{\mu} \frac{\partial}{\partial x^{\mu}} \\
& X_{6}=x_{1} \frac{\partial}{\partial x_{2}}-x_{2} \frac{\partial}{\partial x_{1}}, \\
& X_{7}=x_{3} \frac{\partial}{\partial x_{4}}-x_{4} \frac{\partial}{\partial x_{3}}, \\
& X_{8}=x_{1} \frac{\partial}{\partial x_{3}}-x_{3} \frac{\partial}{\partial x_{1}}-x_{2} \frac{\partial}{\partial x_{4}}+x_{4} \frac{\partial}{\partial x_{2}} \\
& X_{9}=x_{1} \frac{\partial}{\partial x_{4}}-x_{4} \frac{\partial}{\partial x_{1}}+x_{2} \frac{\partial}{\partial x_{3}}-x_{3} \frac{\partial}{\partial x_{2}} \\
& X_{10}=u^{1} \frac{\partial}{\partial u^{1}}+u^{2} \frac{\partial}{\partial u^{2}}, \\
& X_{11}=u^{1} \frac{\partial}{\partial u^{1}}+u^{3} \frac{\partial}{\partial u^{3}} \\
& X_{12}=a(\bar{y}, \bar{z}) \frac{\partial}{\partial u_{2}}
\end{aligned}
$$




$$
\begin{aligned}
& X_{13}=b(y, z) \frac{\partial}{\partial u_{3}}, \\
& X_{14}=f(\bar{y}, \bar{z})\left[u^{1} u^{2} \frac{\partial}{\partial u^{1}}+\left(u^{2}\right)^{2} \frac{\partial}{\partial u^{2}}-\left(u^{1}\right)^{2} \frac{\partial}{\partial u^{3}}\right], \\
& X_{15}=g(y, z)\left[u^{1} u^{3} \frac{\partial}{\partial u^{1}}-\left(u^{1}\right)^{2} \frac{\partial}{\partial u^{2}}+\left(u^{3}\right)^{2} \frac{\partial}{\partial u^{3}}\right],
\end{aligned}
$$

where $a, b$ and $f, g$ are arbitrary functions. Therefore the corresponding space is $L_{\infty}$.

Comparison with the symmetry generators (12) shows that subalgebra $L_{9} \equiv\left\{X_{1}-X_{9}\right\}$ of spatial transformations is the same. The other generators connected with fields are different. Initial field variables can be expressed as

$$
A_{y}^{a}=\frac{1}{u^{1}}\left[i u_{, y}^{2}, u_{, y}^{2},-i u_{, y}^{1}\right], \quad A_{z}^{a}=\frac{1}{u^{1}}\left[i u_{, z}^{3}, u_{, z}^{3},-i u_{, z}^{1}\right] .
$$

Supposing that fields $q^{\alpha}(x)$ in (10) depend only on two coordinates

$$
q^{\alpha}=q^{\alpha}\left(x_{1}, x_{3}\right),
$$

the equation will be

$$
q_{, 11}^{\alpha}+q_{, 33}^{\alpha}+q^{\alpha}\left(q_{, 1}^{\beta} q_{, 1}^{\beta}+q_{, 3}^{\beta} q_{, 3}^{\beta}\right)=0,
$$

which in terms of new variables $w=\frac{1}{2}\left(x_{1}+i x_{3}\right), \bar{w}$ means that

$$
q_{, w w}^{\alpha}+q^{\alpha}\left(q_{, w}^{\beta} q_{, w}^{\beta}\right)=0 .
$$

Imposing the supplementary condition $q^{2}=1$, one can obtain the nonlinear $0(1,3) \sigma$-model $\left[{ }^{15}\right]$. The symmetry group of equations $(15)$ is the following -

$$
\begin{aligned}
& X_{1}=f\left(x_{1}+i x_{3}\right)\left(\frac{\partial}{\partial x_{1}}-i \frac{\partial}{\partial x_{3}}\right), \\
& X_{2}=g\left(x_{1}-i x_{3}\right)\left(\frac{\partial}{\partial x_{1}}+i \frac{\partial}{\partial x_{3}}\right), \\
& X_{\alpha \beta}=q_{\alpha} \frac{\partial}{\partial q_{\beta}}-q_{\beta} \frac{\partial}{\partial q_{\alpha}}
\end{aligned}
$$

where $f$ and $g$ are arbitrary functions.

Generators $X_{1}$ and $X_{2}$ include shifts, rotations and dilatations on the $(1,3)$-plane. Indeed, taking $f=g=1 / 2$ and $f=-g=i / 2$, one gets the shift generators $X_{1}+X_{2}=\frac{\partial}{\partial x_{1}}$ and $X_{1}+X_{2}=\frac{\partial}{\partial x_{3}}$, respectively.

Taking $f(w)=i w, g(\bar{w})=-i \bar{w}$ and $f(w) x=w, g(\bar{w})=\bar{w}$, one obtains the rotation $X_{1}+X_{2}=x_{1} \frac{\partial}{\partial x_{3}}-x_{3} \frac{\partial}{\partial x_{1}}$ and the dilatation $X_{1}+X_{2}=$ $=x_{1} \frac{\partial}{\partial x_{1}}+x_{3} \frac{\partial}{\partial x_{3}}$, respectively. 
In variables $w$ and $\bar{w} X_{1}$ and $X_{2}$ take the forms $X_{1}=f(w) \frac{\partial}{\partial w}$ and $X_{2}=g(\bar{w}) \frac{\partial}{\partial \bar{w}}$.

\section{Conserved quantities}

Here we proceed with the investigation of the considered equations, trying to find some conserved quantities obtainable by means of the Noether theorem $\left[{ }^{20}\right]$. The conserved vector $K_{r}^{\mu}$ satisfies the condition

$$
D_{\mu} K_{r}^{\mu} \equiv \Omega D_{\mu} \xi_{r}^{\mu}+\xi_{r}^{\mu} \frac{\partial \mathfrak{Q}}{\partial x^{\mu}}+\eta_{r}^{a} \frac{\partial \mathfrak{Q}}{\partial u_{\alpha}}+\stackrel{a}{\zeta \mu r}_{\mu r}^{\alpha} \frac{\partial \mathfrak{Q}}{\partial u_{, \mu}^{\alpha}}+\ldots=0 .
$$

Such a vector can be expressed in the form

$$
K_{r}^{\mu}=\left(\eta_{r}^{\alpha}-\stackrel{a}{u_{, v}^{\alpha} \xi_{r}^{v}}\right) \frac{\partial \mathfrak{Q}}{\partial u_{, \mu}^{\alpha}}+2 \xi_{r}^{\mu} \quad(r=1, \ldots, N) .
$$

Here only the equations up to the second order are considered. $\xi_{r}^{v}$ and $\eta_{r}^{\alpha}$ are the coefficients in the $r$-th generator $X_{r}$.

$$
D_{\mu} \equiv \frac{\partial}{\partial x^{\mu}}+\stackrel{a}{u_{, \mu}^{\alpha}} \frac{\partial}{\partial u^{\alpha}}
$$

is the covariant derivative operator.

According to the Noether theorem, the action functional is required to be invariant with respect to an $N$-parameter continuous group of transformations $G$ with a given system of generators $\left\{X_{r}\right\}$. We want to satisfy a weaker condition, i.e. the equation of motion being invariant with respect to a certain group of transformations. In principle, a generator, admitted by the equation of motion, need not leave its action invariant. For this reason one must either check the validity of equation (18) for every generator $X_{r}$, or use a generalization of the Noether theorem $\left[{ }^{8}\right]$ which considers the invariance properties of extremal values of the variational integrals only, instead of regarding all their admissible values.

In case of Yang-Mills equations the knowledge of shift generators $X_{\mu}=\delta_{\mu \nu} \frac{\partial}{\partial x_{v}}$ of symmetry group (5) provides us with a possibility of finding obvious conserved quantities - energy and momentum. Indeed, one can write immediately

$$
D_{\mu} K_{v}^{\mu}=0
$$

and

$$
K_{\mu v}=\stackrel{c}{F}_{\rho \mu} \stackrel{c}{F}_{\rho v}-\frac{1}{4} \delta_{\mu v} \stackrel{c}{F}_{\rho \sigma} \stackrel{c}{F}_{\rho \sigma}
$$

This is the energy-momentum tensor, where the energy density is

$$
H=-K_{11} \quad\left(x^{1} \equiv t\right) .
$$

There is also a conserved quantity-angular momentum tensor

$$
K_{\alpha \beta}^{\mu}=x_{\beta} K_{\alpha}^{\mu}-x_{\alpha} K_{\beta}^{\mu}+\stackrel{c}{F}_{\mu \alpha} \stackrel{c}{A}_{\beta}-\stackrel{c}{F}_{\mu \beta} \stackrel{c}{A}_{\alpha}
$$


for the rotation generator

$$
X_{\alpha \beta}=\xi_{\alpha \beta}^{\mu} \frac{\partial}{\partial x^{\mu}}+\eta_{\alpha \beta}^{\mu} \frac{\partial}{\partial A_{\mu}},
$$

where

$$
\begin{aligned}
& \xi_{\alpha \beta}^{\mu}=\delta_{\mu \alpha} x_{\beta}-\delta_{\mu \beta} x_{\alpha}, \\
& { }_{\eta_{\alpha \beta}}^{\mu}=\delta_{\mu \alpha} A_{\beta}^{c}-\delta_{\mu \beta}{ }^{c} A_{\alpha} .
\end{aligned}
$$

For the Yang equations (13) the Lagrangian is $\left[{ }^{15}\right]-$

$$
\begin{gathered}
\Omega=\frac{1}{2\left(u^{1}\right)^{2}}\left(u_{, y}^{1} u_{, y}^{1}+u_{, z}^{1} u_{, z}^{1}+u_{, y}^{2} u_{, y}^{3}+u_{, z}^{2} u_{, z}^{3}\right)= \\
=\frac{1}{2\left(u^{1}\right)^{2}}\left\{u_{, \mu}^{1} u_{, \mu}^{1}+u_{, \mu}^{2} u_{, \mu}^{3}+i\left(\left[u^{2}, u^{3}\right]_{, 12}+\left[u^{2}, u^{3}\right]_{, 43}\right)\right\},
\end{gathered}
$$

where $\left[u^{2}, u^{3}\right]_{, i k}=u_{, i}^{2} u^{3}{ }_{k}-u_{, k}{ }_{, k} u^{3}$ are the Poisson brackets. Condition $D_{\mu} K_{r}^{\mu}=0$ holds only for conserved quantities which correspond to the generators $X_{v}, X_{6}, X_{7}, X_{8}, X_{9}, X_{12}, X_{13}$. For example,

$$
\begin{aligned}
K_{v}^{\mu}= & \frac{1}{2\left(u^{1}\right)^{2}}\left\{\left(u_{, \rho}^{1} u_{, \rho}^{1}+u_{, \rho}^{2} u_{, \rho}^{3}\right) \delta_{\mu v}-2 u_{, \mu}^{1} u_{, v}^{1}-u_{, \mu}^{2} u_{, v}^{3}-\right. \\
- & u_{, v}^{2} u_{, \mu}^{3}+i \delta_{\mu v}\left(\left[u^{2}, u^{3}\right]_{, 12}+\left[u^{2}, u^{3}\right]_{, 43}\right)+i \delta_{\mu 1}\left[u^{2}, u^{3}\right]_{, 2 v}+ \\
& \left.+i \delta_{\mu 2}\left[u^{2}, u^{3}\right]_{, v 1}+i \delta_{\mu 3}\left[u^{2}, u^{3}\right]_{, v 4}+i \delta_{\mu 4}\left[u^{2}, u^{3}\right]_{, 3 v}\right\} .
\end{aligned}
$$

This is the energy-momentum tensor.

$$
\begin{gathered}
K_{6}^{1}=\frac{1}{2\left(u^{1}\right)^{2}}\left\{x _ { 2 } \left(u_{, 1}^{1} u_{, 1}^{1}+u_{, 1}^{2} u_{, 1}^{3}-\sum_{i=2,3,4}\left(u_{, i}^{1} u_{, i}^{1}+u_{, i}^{2} u_{, i}^{3}\right)+\right.\right. \\
\left.\left.\quad+i\left[u^{2}, u^{3}\right]_{, 34}\right)-x_{1}\left(2 u_{, 1}^{1} u_{, 2}^{1}+\left\{u^{2}, u^{3}\right\}_{, 12}\right)\right\},
\end{gathered}
$$

where $\left\{u^{2}, u^{3}\right\}_{, i k}=u_{, i}^{2} u_{, k}^{3}+u_{, k}^{2} u_{, i}^{3}$;

$$
K_{13}^{\mu}=\frac{b(y, z)}{2\left(u^{1}\right)^{2}}\left[u_{, \mu}^{2}-i\left(u_{, 2}^{2} \delta_{\mu 1}-u_{, 1}^{2} \delta_{\mu 2}-u_{, 4}^{2} \delta_{\mu 3}+u_{, 3}^{2} \delta_{\mu 4}\right)\right] .
$$

The other conserved quantities $K_{6}^{2}, K_{6}^{3}, K_{6}^{4}, K_{7}^{\mu}, K_{8}^{\mu}, K_{9}^{\mu}, K_{12}^{\mu}$ can be obtained analogously by means of equation (19).

Also, for the nonlinear $\sigma$-model equation (15) with the Lagrangian[ $\left.{ }^{15}\right]$

$$
\Omega=\frac{1}{2} \partial_{\mu} q^{\alpha} \partial_{\mu} q^{\alpha} \quad(\mu=1,2)
$$

and with the symmetry group (17) one can obtain conserved vectors $K_{1}^{\mu}, K_{2}^{\mu}, K_{3}^{\mu}$, where

$$
X_{3}=\frac{1}{2} \varepsilon_{\alpha \beta} X_{\alpha \beta} \quad\left(\varepsilon_{\alpha \beta}=-\varepsilon_{\beta \alpha}\right) .
$$

Indeed, $D_{\mu} K_{3}^{\mu}=0$ and 
is the conserved spin-vector.

\section{Conclusions and acknowledgements}

The generators of symmetry groups and several conserved quantities have been found for Yang-Mills equations (at the Lorentz gauge), self-dual Yang-Mills equations and the equations deduced from the latter (Yang equations, nonlinear $\sigma$-model equations, etc.).

Not every generator of an equation is associated with a conserved quantity. For obtaining the conserved currents, the Lagrangian is needed, but, unfortunately, not every equation is provided by the Lagrangian.

In such cases the question of existence and finding the systems of conserved quantities remains open.

We are grateful to $M$. Kõiv for setting the problem and for fruitful discussions, and to A. Ainsaar for support.

\section{REFERENCES}

1. Li e, S., Arch. Math., 6, Heft 3, 328-368 (1881).

2. Lie, S., Engel, F., Theorie der Transformationgruppen, Bd. 1-3, Leipzig, Teubner, $1888,1890,1893$.

3. Tress e, A., Acta Math., $18,1-88$ (1894).

4. Vess i o t, E., Acta Math., 28, 307-349 (1904).

5. Cohen, A., An Introduction to the Lie Theory of. One-Parameter Groups, New York, Stechert, 1931.

6. О в сянни ков Л. В., Лекции по теории групповых свойств дифференциальных уравнений, Новосибирск, «Наука», 1966.

7. И брагимов Н, Х., Групповые свойства некоторых дифференциальных уравнений, Новосибирск, «Наука», 1967.

8. Anderson, R. L., I brag imov, N. H., Lie-Bäcklund transformations in applications, SIAM Philadelphia, 1979.

9. Вл ади м и ров С. А., Группы симметрии дифференциальных уравнений и релятивистские поля, М., Атомиздат. 1979.

10. Proceedings of the Joint IUATM/IMU Symposium. Group theoretical methods in mechanics, USSR, Novosibirsk, 1978.

11. Теоретико-групповые методы в физике. Тр. междунар. семинара, М., «Наука», 1980.

12. Kõ iv, M., A insa a r, A., Ki ir a ne n, K., Preprint F-15, Tartu, 1981.

13. Y a n g, C. N., Mil1s, R. L., Phys. Rev., 96, 191-195 (1954).

14. M a ck, G., S a la m, A., Ann. Phys., 53, № 1, 174-202 (1969).

15. Pohlme yer, K.. Communs Math. Phys., 72, 37-47 (1980).

16. Y a n g, C. N., Phys. Rev. Lett., 38, № 24, 1377-1379 (1977).

17. Forgâcs, P., Horvâth, Z., Palla, L., ITP-Budapest Report N 394 (1980); CRIP preprint, KFKI-1981-06 (1981); CRIP preprint, KFKI-1981-21 (1981).

18. Witt e n, L., Phys. Rev., D19, 718-720 (1979).

19. Corrigan, E., Fa irlie, D. B., Goddard, P., Yates, R. G., Communs Math. Phys., 58, 23-30 (1978).

20. Noether, S., Nachr. Ges. Wiss. Göttingen, Math.-Phys. K1, Heft 2, 235-257 (1918).

Academy of Sciences of the Estonian SSR, Institute of Physics

Received

Nov. 20, 1981 


\section{YANG-MILLSI VORRANDITE JA OMADUAALSE YANG-MILLSI VORRANDI} SUMMEETRIARUHMADEST

Artikkel käsitleb Yang-Millsi võrrandite ja omaduaalse Yang-Millsi võrrandi mõnede kujude poolt lubatud punktteisenduste rühmade leidmise probleemi neljamõõtmelises eukleidilises ruumis. On leitud nende rühmade baasgeneraatorid, samuti mōned jäävad suurused.

\section{B. РОЗЕНГАУЗ, К. КНИРАНЕН \\ О ГРУППАХ СИММЕТРИИ УРАВНЕНИИ ЯНГА-МИЛЛСА И САМОДУАЛЬНОГО УРАВНЕНИЯ ЯНГА-МИЛЛСА}

Рассматривается задача отыскания групп точечных преобразований, допускаемых уравнением Янга-Миллса и некоторыми видами уравнений Янга-Миллса самодуальных полей в четырехмерном евклидовом пространстве. Найден явный вид базисных генераторов данных групп. Получены с помощью теоремы Нётер некоторые сохраняющиеся величины. 